\title{
The Use of Complementary and Alternative Medicine is Frequent in Patients With Pancreatic Disorders
}

\author{
Serena Stigliano, MD, Livia Archibugi, MD, Giulia Zerboni, MD, \\ Gianfranco Delle Fave, MD, and Gabriele Capurso, MD, PhD
}

\begin{abstract}
Background: Herbal remedies and other complementary and alternative medicine (CAM) are used by $30 \%$ of the patients with liver and inflammatory bowel diseases. However, there are no data regarding CAM use in patients with pancreatic disorders, including potential pancreatotoxicity.
\end{abstract}

Aim of the Study: The aim of the study was to assess the prevalence of CAM use in patients with pancreatic disorders and screen for pancreatotoxicity.

Materials and Methods: This was a cross-sectional survey of consecutive outpatients seen at a Pancreas Center. Data were collected in a specific questionnaire. Descriptive statistics were used to analyze the prevalence and the patterns of CAM use. Characteristics associated with CAM use were analyzed by appropriate statistics.

Results: Of 108 patients (52\% male; mean age, $65 \pm 13$ years), 47 $(43.5 \%)$ used CAM. The use of CAM was more frequent among patients with previous acute pancreatitis $(47 \%)$. Reported reasons for the use of CAM were to help standard therapies and for an overall better feeling. About $61 \%$ of the patients reported advantages with treatment. As compared with nonusers, CAM users were more often female $(55 \%$ vs. $42 \%)$, with a higher school degree $(43 \%$ vs. $36 \%)$, more frequently performing physical activity $(51 \%$ vs. $41 \%$ ), and reporting anxiety ( $45 \%$ vs. $31 \%)$. However, none of these differences were statistically significant. Three patients with previous acute pancreatitis reported the use of Serenoa repens that is potentially pancreatotoxic.

Discussion: The rate of CAM use in patients with pancreatic disorders is similar to those reported for other digestive diseases. CAM use seems to be more frequent in women with a higher education level and a "healthier lifestyle." Patients might not be aware of the potential pancreatotoxicity of CAM, which should be carefully considered by physicians.

Key Words: pancreatic disorders, herbal remedies, complementary medicine, pancreatotoxicity

( Clin Gastroenterol 2016;50:S161-S163)

$\mathrm{H}$ erbal remedies and other complementary and alternative medicine (CAM) are frequently used for the management of various conditions including health maintenance, management of anxiety, obesity, diabetes, rheumatologic illness, cancer, cardiovascular disease, and other

From the Digestive and Liver Disease Unit, Sant'Andrea Hospital, Sapienza University, Rome, Italy.

The authors declare that they have nothing to disclose.

Address correspondence to: Gabriele Capurso, MD, PhD, Digestive and Liver Disease Unit, S. Andrea Hospital, Sapienza University of Rome, Via di Grottarossa 1035, Rome 00189, Italy (e-mail: Gabriele.capurso@gmail.com).

Copyright (C) 2016 Wolters Kluwer Health, Inc. All rights reserved.

DOI: $10.1097 /$ MCG.0000000000000685 chronic disorders including those of the gastrointestinal tract. 1

According to the National Institutes of Health, CAM are defined as a group of diverse medical and health care systems, practices, and products that are not presently considered to be part of conventional medicine. ${ }^{2}$

The use of CAM has become widespread among people with chronic disease, as it promotes greater personal control over the health decision, empowers people to manage their chronic condition, and helps avoid the dissatisfaction often associated with conventional health care.

CAM are often considered by individuals using them to be safer than the standard medical practice because they are "natural" or are based on a concept of "wellness" and health. ${ }^{3}$

Previous studies showed that the prevalence of CAM usage ranges from 21 to $60 \%$ in patients with inflammatory bowel $^{4}$ and from $20 \%$ to $50 \%{ }^{1,5}$ in patients with chronic liver disease.

In these previous studies, the most common reason for CAM usage was dissatisfaction with conventional therapy. CAM use seemed more frequent in female patients ${ }^{7}$ and in particular in those with high incomes and a high educational level. ${ }^{5}$

Despite increasing popularity, communication about the use of CAM between physicians and patients is limited, as most physicians know little about CAM and patients avoid discussing about CAM because of the fear of the doctor's disagreement. ${ }^{3}$

Using CAM therapies may be beneficial, but such therapies can also interact with the conventional medications. Most alarmingly, some products may have serious adverse effects, resulting in, for example, a deterioration of the liver function. ${ }^{8}$

Although there are different studies evaluating the prevalence of CAM usage in various chronic gastrointestinal diseases, there are no data regarding CAM usage in patients with pancreatic disorders. In addition, the lack of information is associated with the unawareness of their potential pancreatotoxicity.

\section{AIM}

The primary aim of this study was to assess the prevalence of CAM usage in patients with various pancreatic disorders. A secondary aim was to screen the possible pancreatotoxicity of these products.

\section{MATERIALS AND METHODS}

A cross-sectional study was conducted at a pancreatic disorders outpatient clinic over a 6-month time frame. Consecutive patients with pancreatic disorders were included. 
Data were collected by a trained doctor using a questionnaire regarding demographics and clinical information, CAM usage, and reported reasons for it, the length of usage, and perceived experiences of CAM efficacy. Each participant was also interviewed about their life style, level of education, and current employment status to elicit other relevant exposures. Patients gave an informed consent and the study received local ethic committee approval.

Categorical variables were analyzed by means of the Fisher test and continuous variables expressed by mean \pm SEM were examined by the Student $t$ test. A $P<0.05$ was considered significant. A dedicated software (MedCalc, Mariakerke, Belgium) was used throughout the study.

\section{RESULTS}

One hundred eight consecutive patients were enrolled $(52 \%$ male; mean age, $65 \mathrm{y} \pm 13)$.

The most common reason for the visit at the Pancreatic Disorders Clinic was intraductal papillary mucinous neoplasm (44 patients, $40.7 \%$ ), followed by previous acute pancreatitis (AP) $(34,31.5 \%)$, chronic pancreatitis (20, $19 \%$ ), with other less common disorders such as pancreatic cancer or other cystic lesions in 10 patients $(9.2 \%)$.

Forty-seven of the 108 patients $(44 \%)$ reported the use of CAM: 14 of these $47(29.8 \%)$ patients have used CAM for $>12$ months. The mean age of the "CAM users" was $64 \pm 13$ years, and the majority of them $(55.3 \%)$ were female.

The most frequent reason reported by patients for the use of CAM was to "help standard therapies" $(14,29.7 \%)$ and for an "overall better feeling" $(12,25.5 \%)$. About $61.7 \%$ of the patients reported to have perceived some advantages with treatment.

Only $4 \%$ of the patients who used CAM thought that these products could interfere or interact with the conventional drugs. Furthermore, $28 \%$ of them did not communicate the usage of CAM to their primary-care physician or to other doctors taking care of them.

CAM users more often reported having a high-school or a university degree ( $44 \%$ vs. $36 \%$ of nonusers; $P=0.43$ ) and performing physical activity more than once a week (43\% vs. $34 \% ; P=0.43)$, and were less frequently overweight (body mass index $>2546.8 \%$ vs. $54 \%$; $P=0.56$ ). Regarding other medical disorders, CAM users reported osteomuscular pain $(59.6 \%$ vs. $44,3 \% ; P=0.13)$, anxiety (45\% vs. $31 \% ; P=0.23)$, and symptoms suggestive of irritable bowel syndrome $(17 \%$ vs. $9.8 \% ; P=0.38)$ more frequently as compared with patients not using CAM. However, these differences were not statistically significant (Table 1).

Concerning the category of CAM, $28 \%$ of the 47 CAM users reported the use of herbal remedies, $11 \%$ the use of vitamins, $9 \%$ minerals, and $47 \%$ other nutritional supplements (Table 2).

Regarding the reason for the outpatient visit, the highest rate of CAM users was observed among patients with a previous episode of AP $(16 / 34,47 \%)$, followed by $41 \%(18 / 44)$ in patients with intraductal papillary mucinous neoplasm and $35 \%(7 / 20)$ in patients with chronic pancreatitis.

We also screened the exact composition of the products used for their potential pancreatotoxicity. Interestingly, 3 patients who had previous idiopathic AP have used products composed of Serenoa repens, a substance that has been demonstrated to be associated with pancreatotoxicity before. 9,10
TABLE 1. Demographic and Clinical Features of CAM Users and Nonusers

\begin{tabular}{lccc}
\hline & \multicolumn{3}{c}{$\mathbf{n ~ ( \% )}$} \\
\cline { 2 - 3 } & $\begin{array}{c}\text { CAM Users } \\
\text { Characteristics }\end{array}$ & $\begin{array}{c}\text { Non-CAM Users } \\
\mathbf{( 6 1 )}\end{array}$ & $\boldsymbol{P}$ \\
\hline Sex (female) & $26(55.3)$ & $26(42.6)$ & 0.24 \\
Mean age (y) & 64 & 66 & \\
High education & $21(44)$ & $22(36)$ & 0.43 \\
$\quad$ level & & & \\
BMI <25 & $26(55.3)$ & $28(45.9)$ & 0.56 \\
Smoking & $10(21.3)$ & $17(27.8)$ & 0.51 \\
Osteomuscular & $28(59.6)$ & $27(44.3)$ & 0.13 \\
$\quad$ pain & $8(17)$ & $6(9.8)$ & 0.38 \\
IBS symptoms & $20(42.6)$ & $19(31.2)$ & 0.23 \\
Anxiety &
\end{tabular}

BMI indicates body mass index; CAM, complementary and alternative medicine; IBS, irritable bowel syndrome.

\section{DISCUSSION}

In the last decades, the use of herbal remedies and other nonconventional therapy has increased substantially and has become a factor possibly concerning the management of various chronic gastrointestinal diseases. ${ }^{2,5}$

Previous studies have demonstrated that the use of these products is common in patients with inflammatory bowel disease and chronic liver disease, ${ }^{3,4}$ but there are no data about the prevalence of CAM usage in patients with pancreatic disorders.

We conducted a cross-sectional survey in patients with various pancreatic disorders to assess the rate of usage of these products and to screen for their potential pancreatotoxicity.

Our data showed that $44 \%$ of the patients with pancreatic disorders used CAM, a percentage that is similar to or higher than those reported for other chronic gastrointestinal disorders.

CAM usage was more frequent in patients with previous $\mathrm{AP}$, and in particular, it seemed to be more common in patients with previous idiopathic AP.

In keeping with findings of previous studies, ${ }^{7}$ in the present study, CAM users were more frequently female, with a higher education level and a "healthier lifestyle" compared with nonusers, even if the differences were not statistically significant.

The most common reason for CAM usage was to "help standard therapy" and for an "overall better feeling."

Only a small percentage of patients believed that CAM could interfere with other conventional therapies and not all of them reported the usage to their primary-care physician.

TABLE 2. Most Frequent Categories of Products Used by the 47 Patients Taking CAM

\begin{tabular}{lc}
\hline Category of CAM & No. Patients (\%) \\
\hline Herbal remedies & $13(28)$ \\
Vitamins & $5(11)$ \\
Minerals & $4(9)$ \\
Other nutritional supplements & $22(47)$ \\
\hline
\end{tabular}

CAM indicates complementary and alternative medicine. 
One might hypothesize that this is be because of the general belief that herbal remedies and other nonconventional drugs are safe and natural, and so they cannot have any adverse effects. ${ }^{3}$

However, herbal medicines contain multiple active ingredients that have the potential to be not only beneficial but also harmful under a number of medical conditions. ${ }^{8}$

The effect of herbal medicines may not simply result from the combination of various known active ingredients, but the effect of unrecognized biologically active components of a particular herbal preparation. Because of the complexity of the chemical components and potential actions, a comprehensive understanding of herbal drugs is still challenging. 8

There are few data about the potential toxic effects of CAM on the pancreas. Two case reports showed a possible AP induced by saw-palmetto, a plants that is commonly used in drugs for the treatment of benign prostatic hypertrophy. ${ }^{9}$ This product had been used by 3 patients with previous idiopathic AP in the present survey.

Notably, according to the present data, $45 \%$ of the patients with a previous episode of idiopathic AP reported the use of CAM. These data suggest that the use of potentially pancreatotoxic CAM should be ruled out carefully during the diagnostic work-out of idiopathic AP. ${ }^{10}$

In conclusion, we report for the first time the high prevalence of CAM usage in patients with pancreatic disorders. Physicians should screen their use accurately because patients may not be aware of the potential adverse effects of CAM in this setting.

\section{REFERENCES}

1. Rossi S, Navarro VJ. Herbs and liver injury: a clinical perspective. Clin Gastroenterol Hepatol. 2014;12:1069-1076.

2. Wong AP, Clark AL, Garnett EA, et al. Use of complementary medicine in pediatric patients with inflammatory bowel disease: results from a multicenter survey. $J$ Pediatr Gastroenterol Nutr. 2009;48:55-60.

3. Del Prete A, Scalera A, Iadevaia MD, et al. Herbal products: benefits, limits, and applications in chronic liver disease. Evid Based Complement Alternat Med. 2012;2012:837939.

4. Ng SC, Lam YT, Tsoi KK, et al. Systematic review: the efficacy of herbal therapy in inflammatory bowel disease. Aliment Pharmacol Ther. 2013;38:854-863.

5. Ferrucci LM, Bell BP, Dhotre KB, et al. Complementary and alternative medicine use in chronic liver disease patients. $J$ Clin Gastroenterol. 2010;44:e40-e45.

6. Holtmann G, Talley NJ. Herbal medicines for the treatment of functional and inflammatory bowel disorders. Clin Gastroenterol Hepatol. 2015;13:422-432.

7. Metcalfe A, Williams J, McChesney $J$, et al. Use of complementary and alternative medicine by those with a chronic disease and the general population-results of a national population based survey. BMC Complement Altern Med. 2010;10:58.

8. Nousiainen P, Merras-Salmio L, Aalto K, et al. Complementary and alternative medicine use in adolescents with inflammatory bowel disease and juvenile idiopathic arthritis. $B M C$ Complement Altern Med. 2014;14:124.

9. Bruminhent J, Carrera P, Li Z, et al. Acute pancreatitis with saw palmetto use: a case report. J Med Case Rep. 2011;5:414.

10. Hung WY, Abreu Lanfranco O. Contemporary review of druginduced pancreatitis: a different perspective. World J Gastrointest Pathophysiol. 2014;5:405-415. 